\title{
Discapacidad visual y ceguera entre los estudiantes de una escuela para ciegos en Querétaro, México: una evaluación causal
}

\section{Visual impairment and blindness in students of a school for the blind in Querétaro, México: A causal assessment}

\author{
Rocío Escárcega-Servín ${ }^{1}$, J. Fernando Pérez-Pérez ${ }^{1 *}$, Van Charles Lansingh ${ }^{1,2}$ y Ellery M. Lopez-Star ${ }^{1}$ \\ 1/nstituto Mexicano de Oftalmología IAP, Querétaro, México; ${ }^{2} \mathrm{Help}$ Me See, Nueva York, Estados Unidos
}

\begin{abstract}
Resumen
Objetivo: Describir las causas de ceguera en los alumnos de la escuela para niños ciegos Josefa Vergara en Querétaro, México; así como identificar causas tratables en la población estudiada. Metodología: El estudio se llevó a cabo en el periodo de diciembre de 2017 a febrero de 2018 en la escuela para niños ciegos Josefa Vergara de Querétaro, México. Participaron 30 estudiantes. Los pacientes fueron entrevistados y examinados por los autores. Los resultados obtenidos con el estudio fueron analizados con el programa SPSSMR versión 21. Resultados: La mayoría de los sujetos eran ciegos (28, $93 \%$ ). Las causas más frecuentes de pérdida visual fueron retinopatía del prematuro en 11 pacientes (36.7\%) y catarata congénita en 5 pacientes (16.6\%). En menor porcentaje se encontró amaurosis congénita de Leber, en 2 pacientes (6.7\%), desprendimiento de retina congénito, en 2 pacientes (6.7\%), retinoblastoma, en 2 pacientes (6.7\%), hipoplasia del nervio óptico, en 2 pacientes (6.7\%), entre otras. El 73\% de los participantes en el estudio tenían una causa tratable de ceguera. Conclusiones: La retina fue el principal sitio anatómico afectado. El diagnóstico más común fue secuelas de retinopatía del prematuro. La mayoría de los pacientes tenían alternativas de tratamiento que habrían podido evitar la ceguera en estadios tempranos de su enfermedad. Es necesario implementar y reforzar medidas en el sistema de salud mexicano para la prevención de ceguera en la población pediátrica.
\end{abstract}

Palabras clave: Ceguera. Infantil. Escuela. México. Incidencia. Prevención.

\section{Abstract}

Objective: To describe the causes of blindness in the students of the school for blind children "Josefa Vergara" in Querétaro, Mexico; as well as identifying treatable causes in the studied population. Methodology: The study was conducted in the period from December 2017 to February 2018, at the school for blind children "Josefa Vergara" in Querétaro, Mexico. Thirty students participated. The patients were interviewed and examined by the authors. The results obtained in the study were analyzed with the SPSS ${ }^{T M}$ software version 21. Results: The majority of subjects were blind $28(93 \%)$. The most frequent causes of visual loss were retinopathy of prematurity in 11 patients (36.7\%) and congenital cataract in 5 patients (16.6\%). In

\section{Correspondencia:}

*Fernando Pérez-Pérez

Avenida Estadio, $\mathrm{s} / \mathrm{n}$

Colinas de Cimatario, Centro Sur

Fecha de recepción: 16-10-2018

Fecha de aceptación: 01-04-2019

DOI: 10.24875/RMO.M19000080
Disponible en internet: 01-07-2019 Rev Mex Oftalmol. 2019;93(4):178-184 www.rmo.com.mx 0187-4519/@ 2019 Sociedad Mexicana de Oftalmología. Publicado por Permanyer México. Este es un artículo Open Access bajo la licencia CC BY-NC-ND (http://creativecommons.org/licenses/by-nc-nd/4.0/). 
a lower percentage, Leber's congenital amaurosis was found in 2 patients $(6.7 \%)$, congenital retinal detachment in 2 patients (6.7\%), retinoblastoma in 2 patients (6.7\%), optic nerve hypoplasia in 2 patients (6.7\%), among others. $73 \%$ of the participants in the study had a treatable cause of blindness. Conclusions: The retina was the main anatomical site affected. The most common diagnosis was sequelae of retinopathy of prematurity. Most patients had treatment alternatives that could prevent blindness in early stages of their disease. It is necessary to implement and intensify measures in the Mexican health system for the prevention of blindness in the pediatric population.

Key words: Blindness. Pediatric. School. Mexico. Incidence. Prevention.

\section{Introducción}

A nivel mundial, el número estimado de niños con discapacidad visual es de 19 millones, de los cuales, 12 millones son defectos refractivos no corregidos. Aproximadamente 1.4 millones sufren ceguera irreversible y necesitan acceso a servicios de rehabilitación visual ${ }^{1}$. La prevalencia está en relación con el desarrollo socioeconómico del país, así como con el grado de mortalidad en niños menores de 5 años de edad.

Los resultados de estudios poblacionales indican una menor prevalencia de ceguera en niños que en adultos, y es de aproximadamente 0.2-0.3 por cada 1,000 niños en países industrializados y de 1 a 1-1.5 por cada 1,000 niños en sitios en vías de desarrollo², donde hasta el $75 \%$ de las causas de ceguera en niños son prevenibles o tratables ${ }^{3}$.

Los datos epidemiológicos respecto a prevalencia de ceguera en niños, así como las principales causas, resultan importantes en términos de establecer prioridades para programas de prevención y control. Sin embargo, los distintos tipos de alteraciones resultantes en ceguera varían en incidencia y prevalencia entre regiones y países.

Las principales causas de ceguera infantil pueden identificarse mediante análisis en escuelas para discapacitados visuales 0 a través de estimaciones realizadas por indicadores de salud, por ejemplo, la tasa de mortalidad infantil. Debido a la baja prevalencia de ceguera infantil en la población general, es difícil realizar estudios poblacionales, ya que se requiere un tamaño de muestra grande para tener resultados estadísticos confiables.

Existen múltiples reportes ${ }^{4-6}$ de las causas de ceguera en niños en distintos países de Latinoamérica, producto de estudios realizados en grupos identificables de niños afectados, es decir, en escuelas para niños ciegos. El más reciente de ellos, realizado en Guadalajara, México, en 2011, reporta la retinopatía del prematuro como la principal causa de ceguera con un porcentaje de $34.7 \% 4$.

Los estudios epidemiológicos reportan la opacidad corneal como causa más común de ceguera infantil en sitios con alta tasa de mortalidad infantil, como es
Bolivia, República Dominicana y Perú, donde se le puede atribuir alrededor del $20 \%$ de las causas. En contraste con países como EE.UU., Chile y Argentina, que reportan muy pocos $\operatorname{casos}^{5,6}$.

La retinopatía del prematuro es una importante causa de ceguera en países con mortalidad intermedia, donde la tasa de supervivencia de recién nacidos con bajo peso ha incrementado, pero persisten las deficiencias en las pautas de cuidado neonatal. Tal es el caso de países como Cuba, México y Paraguay, donde representa el $30 \%$ de las causas de ceguera. En contraste con EE.UU., con una frecuencia del $8 \%$, probablemente como resultado del adecuado cuidado intensivo neonatal. Así mismo, Bolivia, República Dominicana y Perú reportan la retinopatía del prematuro como una causa poco frecuente, muy posiblemente por la baja tasa de sobrevida de los prematuros ${ }^{5,6}$.

La catarata presenta una prevalencia homogénea entre las distintas regiones y se encuentra entre las principales causas de ceguera reportadas en la literatura, esta puede ser de origen congénito 0 adquirido, relacionada con la rubeola y con enfermedades genéticas ${ }^{5,6}$.

El programa de la Organización Mundial de la Salud para la prevención de ceguera y el International Centre for Eye Health desarrollaron una metodología estandarizada para el reporte de las causas de pérdida visual en niños, con especial énfasis en la identificación de las causas prevenibles o tratables ${ }^{7}$.

La clasificación anatómica de las causas de pérdida visual define la parte del ojo que se encuentra dañada y que condiciona la mayor pérdida visual. Cuando dos 0 más sitios anatómicos estuvieron involucrados, se selecciona el mayor, y si contribuyen de igual manera, se selecciona la condición más tratable. La clasificación etiológica se divide en cinco categorías, según el tiempo de aparición de la condición que llevó a la ceguera (hereditaria, intrauterina, perinatal, infancia y desconocido) ${ }^{7}$.

La Organización Mundial de la Salud, de acuerdo con la Clasificación Internacional de Enfermedades (CIE-10), define como déficit visual severo aquellos pacientes con una agudeza visual corregida menor a 20/200 en el mejor ojo, pero mejor de 10/200, y como ceguera, una 
agudeza visual peor de que 20/400 en el mejor ojo. Es importante hacer la distinción entre ambas entidades, ya que las personas con déficit visual, a diferencia de aquellas con ceguera, conservan todavía un resto de visión funcional, útil para desenvolverse en la vida diaria ${ }^{8}$.

La ceguera pediátrica representa un problema en términos de morbilidad, pérdida económica y carga social, ya que afecta los años de edad productiva laboral. En contraste con los adultos, que, de forma general, se vuelven ciegos en edades más tardías, con una repercusión social menor.

México tiene una población de más de 120 millones de habitantes, se encuentra en el lugar 56 en el Programa de Desarrollo de las Naciones Unidas y es clasificado como un país con alto nivel de desarrollo. En 2015, la expectativa de vida era de 76.92 años y el $98 \%$ de los niños estaban inscritos a un programa de educación primaria. Querétaro es uno de los 32 estados de México, tiene una población de 2,038,372, de los cuales, 545,065 son niños, es decir, el $30 \%$ de los habitantes del estado ${ }^{9}$. La tasa de mortalidad infantil en Querétaro es de 14 por cada 1,000 nacimientos, de acuerdo al Instituto Nacional de Estadística, Geografía e Informática (INEGI) en 2011. Ambos índices sugieren que la prevalencia de ceguera en niños es baja y que la retinopatía del prematuro puede ser una causa importante, sin embargo, hay una escasa información de las causas de ceguera infantil en México.

La prevención de ceguera por retinopatía del prematuro requiere de un cuidado neonatal de calidad, así como de programas que garanticen la detección oportuna. Las guías para prevención, detección y tratamiento de retinopatía del prematuro fueron publicadas en México en 2007, pero no están ampliamente implementadas.

\section{Objetivo}

Describir las causas de ceguera en los alumnos de la escuela para niños ciegos Josefa Vergara en Querétaro, México, así como identificar causas tratables en la población estudiada.

\section{Metodología}

El estudio fue diseñado para determinar las causas de ceguera entre los estudiantes de la escuela para niños ciegos en Querétaro, México, así como identificar causas tratables de ceguera en la población estudiada.

Se visitó el centro educativo para niños ciegos Josefa Vergara en el período entre diciembre de 2017 a febrero de 2018 en Querétaro, México. Participaron los
30 estudiantes inscritos en esta escuela, con un rango de edad de 4 a 18 años. Se obtuvo aprobación para el estudio por parte de las autoridades de la escuela, la firma de consentimiento informado de los padres o tutores y la aprobación del comité de ética y de investigación del IMO. Los pacientes y sus padres fueron entrevistados por los autores.

Todos los estudiantes fueron examinados por un grupo de oftalmólogos y un oftalmólogo pediatra. Se obtuvo información respecto a diagnósticos oftalmológicos y sistémicos previos, cirugías y tratamientos, valoración de estudios de gabinete anteriores, agudeza visual al momento del estudio, toma de refracción en caso necesario y exploración oftalmológica.

Se siguió el protocolo clínico para la medición de la agudeza visual lejana en pacientes con baja visión, propuesto por la Academia Americana de Oftalmología, en donde, si el paciente no puede distinguir el optotipo a la distancia establecida, se acerca progresivamente la cartilla hasta obtener una agudeza visual medible. Se utilizaron las cartillas de Snellen y E iletrada. En pacientes en los que mejoraba la agudeza visual con estenopeico o tenían una agudeza visual mejor a percepción de luz sin déficit anatómico significativo, se realizó refracción con retinoscopía y regletas.

Respecto a la exploración oftalmológica, el estudio del segmento anterior se hizo con lámpara de hendidura portátil y el del segmento posterior, mediante oftalmoscopía directa. En los pacientes que lo requirieron, se realizó dilatación farmacológica y exploración con oftalmoscopio indirecto y lentes de $20 \mathrm{D}$ y $28 \mathrm{D}$.

Se siguió la guía del programa de la Organización Mundial de la Salud para la prevención de ceguera y el del International Centre for Eye Health ${ }^{8}$ para el reporte de las causas de pérdida visual. Posterior al registro de todas las anormalidades en cada ojo, se eligió un sitio anatómico como el principal causante de ceguera. La etiología subyacente se determinó, en los casos en que fue posible, y se categorizó de acuerdo con el tiempo de aparición de la condición.

En cuanto al pronóstico visual, se siguieron los rangos de agudeza visual propuestos por la Organización Mundial de la Salud y la Clasificación Internacional de Enfermedades, CIE-10. Se definió como buen pronóstico visual aquellos que con corrección óptica alcancen una capacidad visual de 20/60 en el mejor ojo, pronóstico visual moderado, aquellos en rango de 20/80 a 20/200, y mal pronóstico, aquellos con capacidad visual peor a 20/200 en el mejor ojo.

A todos los pacientes se les dio referencia al centro oftalmológico Instituto Mexicano de Oftalmología 
Table 1. Edad

\begin{tabular}{|c|c|c|}
\hline Anos & Frecuencia & Porcentaje \\
\hline 4 & 1 & $3.3 \%$ \\
\hline 5 & 2 & $6.7 \%$ \\
\hline 7 & 4 & $13.3 \%$ \\
\hline 8 & 3 & $10.0 \%$ \\
\hline 9 & 3 & $10.0 \%$ \\
\hline 10 & 5 & $16.7 \%$ \\
\hline 11 & 2 & $6.7 \%$ \\
\hline 12 & 4 & $13.3 \%$ \\
\hline 13 & 1 & $3.3 \%$ \\
\hline 14 & 1 & $3.3 \%$ \\
\hline 16 & 2 & $6.7 \%$ \\
\hline 18 & 2 & $6.7 \%$ \\
\hline Total & 30 & $100.0 \%$ \\
\hline
\end{tabular}

(Instituto de Asistencia Privada, IAP) para abrir su expediente y dar seguimiento a su padecimiento oftalmológico. Algunos de ellos necesitaban estudios y valoraciones adicionales, con la finalidad de determinar la etiología o brindar el tratamiento. Los resultados obtenidos fueron recabados, procesados y analizados con el software estadístico SPSS ${ }^{\text {MR }}$ versión 21.

\section{Resultados}

Se estudiaron 30 pacientes de la escuela para niños ciegos Josefa Vergara en Querétaro, México. En un rango de edad de 4 a 18 años (Tabla 1), 20 mujeres (66.7\%) y 10 hombres (33.3\%). La mayoría de los sujetos eran ciegos $(28[93 \%])$ y $2(7 \%)$ tenían visión baja. De los pacientes legalmente ciegos, 14 (46.6\%) presentaban visión de no percepción de luz, 2 (7\%) percepción de luz, $6(20 \%)$ movimiento de manos, $4(13 \%)$ en contar dedos a un metro, y 2 (7\%) en 20/200. La mayoría de los pacientes no tenían historia de cirugía ocular (26 [86.7\%]), mientras que 4 (13.3\%) tenían algún antecedente quirúrgico.

Las causas más frecuentes de pérdida visual fueron secuelas de retinopatía del prematuro en 11 pacientes $(36.7 \%)$ y catarata congénita en 5 pacientes (16.6\%). Otras causas encontradas en menor porcentaje fueron amaurosis congénita de Leber, en 2 pacientes (6.7\%), desprendimiento de retina congénito, en 2 pacientes (6.7\%), retinoblastoma, en 2 pacientes $(6.7 \%)$,
Table 2. Causa principal de déficit visual

\begin{tabular}{|l|c|c|}
\hline Diagnóstico & $\begin{array}{c}\text { Número de } \\
\text { pacientes }\end{array}$ & Porcentaje \\
\hline ROP & 11 & $36.7 \%$ \\
\hline Catarata congénita & 5 & $16.6 \%$ \\
\hline Amaurosis congénita de Leber & 2 & $6.7 \%$ \\
\hline Hipoplasia del nervio óptico & 2 & $6.7 \%$ \\
\hline Retinoblastoma & 2 & $6.7 \%$ \\
\hline Desprendimiento de retina congénito & 2 & $6.7 \%$ \\
\hline Glaucoma por aniridia & 1 & $3.3 \%$ \\
\hline Distrofia retiniana no especificada & 1 & $3.3 \%$ \\
\hline Ectopia lentis & 1 & $3.3 \%$ \\
\hline Ametropía & 1 & $3.3 \%$ \\
\hline Leucoma corneal & 1 & $3.3 . \%$ \\
\hline Atrofia del nervio óptico & 1 & $3.3 \%$ \\
\hline
\end{tabular}

hipoplasia del nervio óptico, en 2 pacientes (6.7\%), entre otras, (Tabla 2). Acerca de los factores sistémicos asociados se encontraron el síndrome de TORCH y el síndrome de Marfán, un paciente con cada alteración, así como un caso de astrocitoma subependimario de células gigantes resecado (Tabla 3 ).

La retina fue el principal sitio anatómico de pérdida visual, con el $60 \%$, como se detalla en la tabla 4 , seguido del cristalino, en tercer lugar, el nervio óptico y, en menor porcentaje, la córnea y todo el globo ocular, con el $3.3 \%$ cada uno.

En cuanto a los factores etiológicos (Tabla 5), en primer lugar se encontraron los intrauterinos y los perinatales, con el $36.7 \%$, en ambos casos, y en menor porcentaje se encontraron factores presentados en la infancia, y en el $6.7 \%$ de los pacientes eran desconocidos.

El $73 \%$ de los participantes del estudio tenían una causa de ceguera que pudo ser tratable en estadios tempranos de la enfermedad o incluso evitable. En cuanto al pronóstico de restauración de la visión, la mayoría de los pacientes, 28 (93\%), tenía un pobre pronóstico, y 2 pacientes (6.7\%) tenían un pronóstico moderado de restauración de la agudeza visual. Ninguno de los individuos tenía buen pronóstico visual.

Los resultados de este estudio muestran distintas causas de ceguera con origen en cada uno de los sitios anatómicos oculares y con múltiples alteraciones asociadas. La presencia de nistagmo se encontró en 13 
Table 3. Asociaciones sistémicas y hallazgos

\begin{tabular}{|l|c|l|}
\hline Alteración & Pacientes & Hallazgos oftalmológicos \\
\hline TORCH & 1 & $\begin{array}{l}\text { Ojo derecho catarata total } \\
\text { Ojo izquierdo retina en sal } \\
\text { y pimienta, hipoplasia del } \\
\text { nervio óptico }\end{array}$ \\
\hline $\begin{array}{l}\text { Síndrome de } \\
\text { Marfán }\end{array}$ & 1 & $\begin{array}{l}\text { Ojo derecho ectopia lentis } \\
\text { Ojo izquierdo afaquia } \\
\text { quirúrgica }\end{array}$ \\
\hline $\begin{array}{l}\text { Astrocitoma } \\
\text { subependimario de } \\
\text { células gigantes } \\
\text { resecado }\end{array}$ & 1 & $\begin{array}{l}\text { Ambos ojos atrofia del } \\
\text { nervio óptico }\end{array}$ \\
\hline
\end{tabular}

Tabla 4. Sitio anatómico de pérdida visual

\begin{tabular}{|l|c|c|}
\hline Sitio anatómico & Frecuencia & Porcentaje \\
\hline Retina & 18 & $60 \%$ \\
\hline Cristalino & 6 & $20 \%$ \\
\hline Nervio óptico & 4 & $13.3 \%$ \\
\hline Córnea & 1 & $3.3 \%$ \\
\hline Todo el globo & 1 & $3.3 \%$ \\
\hline
\end{tabular}

pacientes (43.3\%), y $8(26.7 \%)$ tuvieron también estrabismo (Tablas 6 y 7 ).

\section{Discusión}

La mayoría de los estudios reportados en la literatura con la misma rama de análisis y metodología, es decir, estudios en escuelas para niños ciegos, reportan resultados muy similares. La proporción de pacientes con déficit visual importante es muy elevada, $93 \%$ en este ensayo. Un estudio en Etiopía reporta un $94 \%$ de los estudiantes con ceguera ${ }^{10}$, otro, realizado en Edimburgo, reporta ceguera en el $81 \%$ de los estudiantes ${ }^{11}$. Nuestros resultados también son consistentes con un estudio hindú en el que el $91.7 \%$ de los sujetos fueron clasificados como ciegos $^{12}$. Es destacable en esta situación, que se encuentran inscritos niños que no son ciegos en escuelas destinadas únicamente a este sector poblacional.

La retinopatía del prematuro fue la causa más frecuente de déficit visual en nuestra población, con el $36.7 \%$, y la retina fue el principal sitio anatómico afectado, en contraste con otros estudios que reportan al cristalino como el principal sitio afectado, que en este caso ocupó el segundo lugar, con el $20 \%$. Un estudio de Nigeria reporta que el cristalino fue el $30.7 \%$ y la
Tabla 5. Etiología de ceguera y discapacidad visual

\begin{tabular}{|l|c|c|}
\hline Etiología & Frecuecia & Porcentaje \\
\hline Hereditaria & 4 & $13.3 \%$ \\
\hline Intrauterina & 11 & $36.7 \%$ \\
\hline Perinatal & 11 & $36.7 \%$ \\
\hline Infancia & 2 & $6.7 \%$ \\
\hline Desconocido & 2 & $6.7 \%$ \\
\hline
\end{tabular}

\section{Table 6. Nistagmus}

\begin{tabular}{|l|c|c|}
\hline & Frecuencia & Porcentaje \\
\hline No & 17 & $56.7 \%$ \\
\hline Sí & 13 & $43.3 \%$ \\
\hline Total & 30 & $100.0 \%$ \\
\hline
\end{tabular}

Table 7. Estrabismo

\begin{tabular}{|l|c|c|}
\hline & Frecuencia & Porcentaje \\
\hline No & 22 & $73.3 \%$ \\
\hline Sí & 8 & $26.7 \%$ \\
\hline Total & 30 & $100.0 \%$ \\
\hline
\end{tabular}

principal de las causas ${ }^{13}$. Sin embargo, existe un estudio realizado en India que le atribuye el $31.1 \%$ a la retina como principal sitio afectado ${ }^{9}$ y otro en la República Checa que nos habla del $54.2 \%{ }^{14}$, muy similar a lo encontrado en nuestro estudio, que fue del $60 \%$, pero las causas que nos reportan ambos estudios son de distrofias retinianas o albinismo y apenas un 1.9\% debido a retinopatía del prematuro ${ }^{15}$, en contraste con lo encontrado en literatura latinoamericana con resultados concordantes a los de la muestra de este estudio en países con desarrollo similar a México, como Argentina, Paraguay y Cuba, con el $30 \%$ de las casusas atribuibles a retinopatía del prematuro ${ }^{5,6}$.

En artículos científicos previos, reportan al sarampión como una de las principales causas ${ }^{12}$, sin embargo, la inmunización implementada en la cartilla de vacunación en México desde 1978 puede correlacionarse con la incidencia baja en este estudio, ya que no hay brotes reportados desde $1996^{16} \mathrm{y}$, por lo tanto, la incidencia del factor etiológico en la infancia es muy baja.

La mayoría de los pacientes en este estudio no tuvieron revisiones oftalmológicas para la detección de enfermedades oftalmológicas y, por lo tanto, no se les ofreció cirugía o tratamiento en etapas tempranas de 
su enfermedad. A diferencia de lo reportado en la literatura, en la que a la mayoría de los pacientes tampoco se les ofrece cirugía, pero por tratarse de una condición no quirúrgica ${ }^{3}$.

El ofrecer cirugía en esta etapa se asociaría con pobre pronóstico, relacionado con el tiempo de evolución y el mal desarrollo de la función foveal. Esto pone de manifiesto la importancia de un pronto diagnóstico y tratamiento de las enfermedades oftalmológicas.

En México se cuenta con los lineamientos para la detección y tratamiento de la retinopatía del prematuro desde 2007, que indican valoración oftalmológica, especialmente de retina, a todo paciente prematuro nacido de menos de 34 semanas de gestación y/o con peso al nacimiento menor o igual a $1,750 \mathrm{~g}$, así como prematuros por encima de estos parámetros, a criterio del médico tratante, por factores de riesgo asociados o evolución clínica ${ }^{17}$. En el año 2013 se modificó la Ley General de Salud con la finalidad de que se cumplan estos lineamientos, obligando a la revisión de retina del paciente prematuro en la cuarta semana de vida extrauterina ${ }^{18}$.

Congruente con lo reportado en la literatura, el 73\% de los pacientes tenían una causa de ceguera tratable con distintos pronósticos visuales de acuerdo al tipo y grado de alteración si hubiera sido detectada en estadios tempranos. Previamente se comentó que en países en vías de desarrollo esta cifra se encuentra en el $75 \%$ de los casos 8 .

Dos de los pacientes eran débiles visuales y no ciegos, por lo que su método de enseñanza y rehabilitación podrían ser distintos. Uno de los pacientes tenía diagnóstico de ametropía con pronóstico visual moderado al momento del estudio, por lo que se indicó corrección óptica con lentes aéreos y seguimiento estrecho para su posible reubicación en otra clase de escuela. Es muy importante analizar un posible tratamiento para todos los niños con déficit visual antes de su inscripción a una escuela para ciegos.

Es difícil disponer de información confiable de las causas de pérdida visual en niños cuando no se cuenta con un sistema de registro de ceguera. El examinar niños en escuelas para ciegos puede proveer esta información de manera relativamente fácil, pero limitada por distintos factores, y no puede usarse para calcular datos de prevalencia específicos.

La prevalencia estimada de ceguera pediátrica en México es de 0.3 en 1,000 niños ${ }^{19}$, calculada mediante el modelo propuesto por Gilbert y Foster ${ }^{20}$ basándose en la mortalidad infantil en menores de 5 años. De acuerdo a esta prevalencia, hay aproximadamente 164 niños con ceguera en Querétaro, por lo que en este grupo tuvo una muestra de aproximadamente el $18 \%$ de ellos.

Es importante considerar que no todos los niños ciegos tienen acceso a la educación especial, ni son aceptados en estas escuelas, por ejemplo, aquellos que tienen alguna discapacidad adicional, como retraso en el desarrollo psicomotor, no van a estar representados en esta muestra, al igual que los niños preescolares. Sin embargo, la obtención de datos de estos grupos poblacionales es difícil de obtener, ya que no están inscritos en ningún programa de rehabilitación o distribuidos de manera aleatoria en las distintas escuelas para niños con retraso psicomotor.

La asociación con nistagmo y estrabismo son esperadas por tratarse de pacientes con déficit visual importante y ceguera, sin embargo, se encontró una prevalencia mucho más alta que la reportada en la literatura, con un $43.3 \%$ y $26.7 \%$, respectivamente. Distintos estudios hablan de una prevalencia del $11.9 \%$ para nistagmo y del $20-28 \%$ para estrabismo ${ }^{21-23}$.

\section{Conclusiones}

Este estudio, aunque se trate de una muestra pequeña, proporciona un reflejo de las alteraciones prevalentes en nuestra población para ceguera en niños y pone de manifiesto la necesidad de implementar y reforzar medidas en el sistema de salud para la prevención de ceguera en población pediátrica. Tomando en cuenta las repercusiones con relación a salud y calidad de vida, pero también económicas y sociales, debería convertirse en una prioridad de atención, ya que el $73 \%$ de las causas de ceguera de este estudio eran evitables. La retina fue el sitio principal anatómico afectado y causante de ceguera y discapacidad visual, seguido del cristalino. El diagnóstico más común fue retinopatía del prematuro, seguido de catarata. Todo ello resalta la importancia de una revisión oftalmológica desde el primer mes de vida.

\section{Responsabilidades éticas}

Protección de personas y animales. Los autores declaran que para esta investigación no se han realizado experimentos en seres humanos ni en animales.

Confidencialidad de los datos. Los autores declaran que han seguido los protocolos de su centro de trabajo sobre la publicación de datos de pacientes.

Derecho a la privacidad y consentimiento informado. Los autores declaran que en este artículo no aparecen datos de pacientes. 


\section{Bibliografía}

1. World Health Organization, Global Data on Visual Impairments 2010 Geneve: WHO; 2012. Disponible en: http://www.who.int/blindness/GLOBALDATAFINALforweb.pdf. Consultado el 22 de marzo 2018.

2. Gilbert CE, Anderton L, Dandona L, Foster A. Prevalence of visual impairment in children: a review of available data. Ophthalmic Epidemiol. 1999;6(1):73-82.

3. Omolase CO, Aina AS, Omolase BO, Omolade EO. Causes of blindness and visual impairment at the school for the blind owo, Nigeria. Ann Ib Postgrad Med. 2008:6(1):49-52.

4. Zepeda-Romero LC, Barrera-de-Leon JC, Camacho-Choza C, Gonzalez Bernal C, Camarena-Garcia E, Diaz-Alatorre C, et al. Retinopathy of prematurity as a major cause of severe visual impairment and blindness in children in schools for the blind in Guadalajara City, Mexico. $\mathrm{Br}$ Ophthalmol. 2011;95:1502-5

5. Gilbert C, Fielder A, Gordillo L. Quinn G, Semiglia R, Visintin R, Zin A Characteristics of Infants with severe retinopathy of prematurity in countries with low, moderate and high levels of development: implications for screening programs. Pediatrics. 2005;115(5):e518-25.

6. Muñoz B, West SK. Blindness and visual impairment in the Americas and the Caribbean. Br J Ophthalmol. 2002;86:498-504

7. Gilbert C, Foster A, Negrel AD, Thylefors B. Childhood blindness: a new form for recording causes of visual loss in children. Bull World Health Organ. 1993;71:485-9.

8. Organización Mundial de la Salud. CIE-10. Enfermedades del ojo y anexos (HOO-H59). Cap. VII; 2016.

9. Senso de población y vivienda INEGI, información por entidad. Disponible en: http://cuentame.inegi.org.mx/monografias/informacion/queret/poblacion/default.aspx?tema $=$ me\&e $=22$

10. Kello $A B$, Gilbert $C$. Causes of severe visual impairment and blindness in schools for the blind in Ethiopia. Br J Ophthalmol. 2003:87:526-30.

11. Alagaratnam J, Sharma TK, Lim CS, Fleck BW. A survey of visual impairment in children attending the Royal Blind School, Edinburg using the WHO childhood visual impairment data base. Eye. 2002;16(5):557-61
12. Hornby SJ, Adolph S, Gothwal VK, Gilbert CE, Dandona L, Foster A. Evaluation of children in six blind schools of Andhra Pradesh. Indian J Ophthalmol. 2000;48:195-200.

13. Ezegwu IR, Umeh RE, Ezepue UF. Causes of childhood blindness: results from school for the blind in South Eastern Nigeria. Br J Ophthalmol. 2003;87(1):20-3.

14. Kocur I, Kuchynka P, Rodny S, Barakova D, Schwartz EC. Causes of severe visual impairment and blindness in children attending schools for the visually handicapped in Czech Republic.Br J Ophthalmol. 2001:85:1149-52

15. Hornby SJ, Xiao Y, Gilbert CE, Foster A, Wang X, Liang X, et al. Causes of childhood blindness in the People's Republic of China: results from 1131 blind school students in 18 provinces. Br J Ophthalmol. 1999;83 (8):929-32.

16. Consejo Nacional de Vacunación. Programa de Vacunación Universal 1995-2000. México, DF: CONAVA; 1995.

17. Organización Panamericana de la Salud. Pautas para el examen, detección y tratamiento, de la retinopatía del prematuro en Latinoamérica. Disponible en: http://www.v2020la.org/docs/guias rop cancun 07.pdf

18. Secretaria de Salud de México. Manejo de la retinopatia del recién nacido prematuro. Lineamiento técnico. 2. ${ }^{a}$ ed. 2010. ISBN-978-970-721-441-5 (citado 3 de agosto de 2015). Disponible en: http://saludmaternamedicos. blogspot.mx/2011/09/lineamiento-tecnico-manejo-de-la.html

19. Gilbert C, Awan H. Blindness in children. BMJ. 2003;327 (7418):760.

20. Foster A, Gilbert CE. Epidemiology of childhood blindness. Eye. 1992; 6:173-6.

21. Fakhoury O, Aziz A, Matonti F, Benso C, Belahda K, Denis D. [Epidemiologic and etiological characteristics of congenital cataract: study of 59 cases over 10 years] J Fr Ophtalmol. 2015;38:295-300.

22. Kim KH, Ahn K, Chung ES, Chung TY. Clinical outcomes of surgical techniques in congenital cataracts. Korean J Ophthalmol. 2008:22:87-91.

23. Zhu X, Du Y, He W, Sun T, Zhang Y, Chang R, et al. Clinical Features of Congenital and Developmental Cataract in East China: A Five-year Retrospective Review. Sci Rep. 2017;26;7(1):4254. 